\title{
Elliptic Flow from a Hybrid CGC, Full 3D Hydro and Hadronic Cascade Model
}

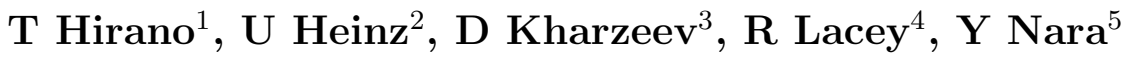 \\ ${ }^{1}$ Department of Physics, the University of Tokyo, Tokyo 113-0033, Japan \\ ${ }^{2}$ Department of Physics, Ohio State University, Columbus, OH 43210, USA \\ ${ }^{3}$ Physics Department, Brookhaven National Laboratory, Upton, NY 11973-5000, USA \\ ${ }^{4}$ Department of Chemistry, SUNY Stony Brook, Stony Brook, NY 11794-3400, USA \\ ${ }^{5}$ Institut für Theoretische Physik, J. W. Goethe-Universität, Max v. Laue Str. 1, \\ D-60438 Frankfurt, Germany \\ E-mail: hirano@phys.s.u-tokyo.ac.jp
}

\begin{abstract}
We investigate the robustness of the discovery of the perfect fluid through comparison of hydrodynamic calculations with the elliptic flow coefficient $v_{2}$ at midrapidity in $\mathrm{Au}+\mathrm{Au}$ collisions at $\sqrt{s_{N N}}=200 \mathrm{GeV}$. Employing the Glauber model for initial entropy density distributions, the centrality dependence of $v_{2}$ is reasonably reproduced by using an ideal fluid description of the early QGP stage followed by a hadronic cascade in the late hadronic stage. On the other hand, initial conditions based on the Colour Glass Condensate model are found to generate larger elliptic flow due to larger initial eccentricity $\varepsilon$. We further predict $v_{2} / \varepsilon$ at a fixed impact parameter as a function of collision energy $\sqrt{s_{N N}}$ up to the LHC energy.
\end{abstract}

One of the important discoveries made at the Relativistic Heavy Ion Collider (RHIC) is the large elliptic flow $v_{2}$ in non-central $\mathrm{Au}+\mathrm{Au}$ collisions [1]. At the highest RHIC energy, the observed $v_{2}$ values near midrapidity at low transverse momentum $\left(p_{T}\right)$ in central and semicentral collisions agree with predictions from ideal fluid dynamics [2]. The ideal fluid dynamical description gradually breaks down, however, as one moves away from midrapidity or studies peripheral collisions. This requires a more realistic treatment of the early and late stages in dynamical modeling of relativistic heavy ion collisions.

We have formulated a dynamical and unified model [3], based on fully threedimensional (3D) ideal fluid dynamics [4, 5], toward understanding the bulk and transport properties of the quark gluon plasma (QGP). During the fluid dynamical evolution we assume local thermal equilibrium. However, this assumption can be expected to hold only during the intermediate stage of the collision. In order to extract properties of the QGP from experimental data one must therefore supplement the hydrodynamic description by appropriate models for the beginning and end of the collision. For the early stage, we employ the Colour Glass Condensate (CGC) picture for colliding nuclei and calculate the produced gluon distributions as input for the initial conditions in the fluid dynamical calculation [6]. During the late stage, local thermal 

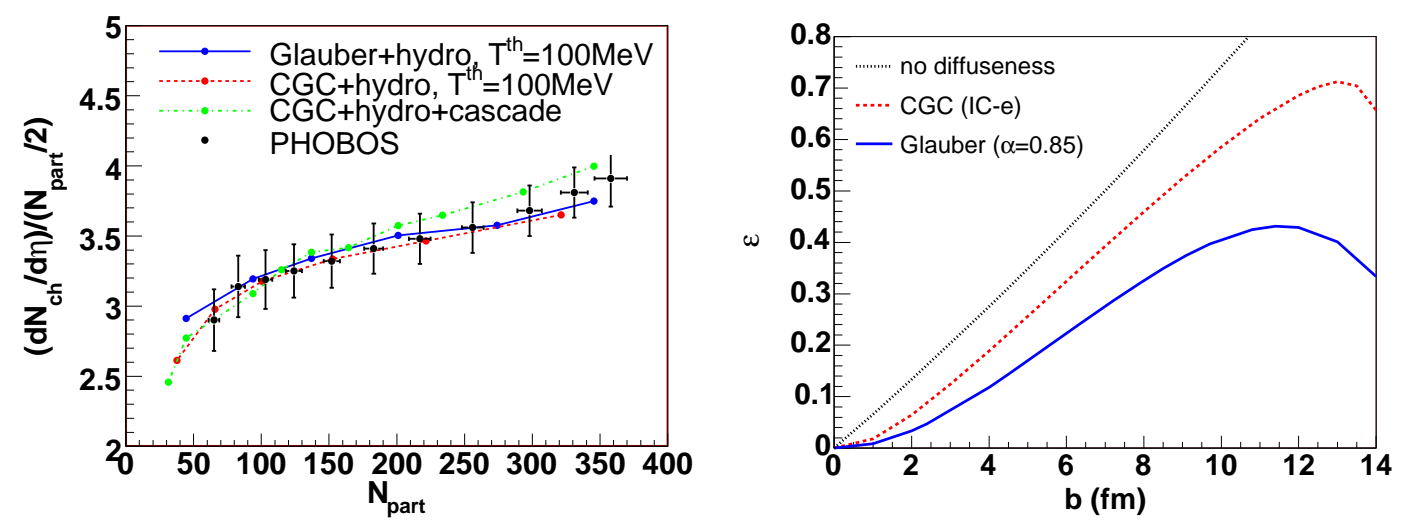

Figure 1. Centrality dependence of charged particle multiplicity per number of participant nucleons (left) and impact parameter dependence of the eccentricity of the initial energy density distributions (right). The solid (dashed) line results from Glauber-type (CGC) initial conditions. The dash-dotted line in the left figure results from our hybrid model. Experimental data are from PHOBOS [8. The dotted line in the right figure assumes a box profile for the initial energy density distribution.

equilibrium is no longer maintained due to expansion and dilution of the matter. We treat this gradual transition from a locally thermalised system to free-streaming hadrons via a dilute interacting hadronic gas by employing a hadronic cascade model [7].

We first calculate the centrality dependence of the multiplicity to see that the CGC indeed correctly describes the initial entropy production and gives proper initial conditions for the fluid dynamical calculations. For reference we compare with the conventional approach where the initial entropy density is parametrized as a superposition of terms scaling with the densities of participant nucleons and binary collisions from the Glauber model. Both CGC and Glauber model initial conditions, propagated with ideal fluid dynamics, reproduce the observed centrality dependence of the multiplicity [8], see Fig. 1] (left). This agreement with the data still holds when the ideal fluid description is replaced by a more realistic hadronic cascade below a switching temperature of $T^{\mathrm{sw}}=169 \mathrm{MeV}$. In the right panel of Fig. 1 1 we show the impact parameter dependence of the eccentricity of the initial energy density distributions. We neglect event-by-event eccentricity fluctuations although these might be important for very central and peripheral events [9]. Even though both models correctly describe the centrality dependence of the multiplicity, they exhibit a significant difference: The eccentricity from the CGC is 20-30\% larger than that from the Glauber model [10]. The initial eccentricity is thus quite sensitive to model assumptions about the initial energy deposition which can be discriminated by the observation of elliptic flow.

With Glauber model initial conditions [11], the predicted $v_{2}$ from ideal fluid dynamics overshoots the peripheral collision data [12]. Hadronic dissipative effects within hadron cascade model reduce $v_{2}$ and, in the Glauber model case, are seen to be sufficient to explain the data (Fig. 2 (left)) [3]. Initial conditions based on the 

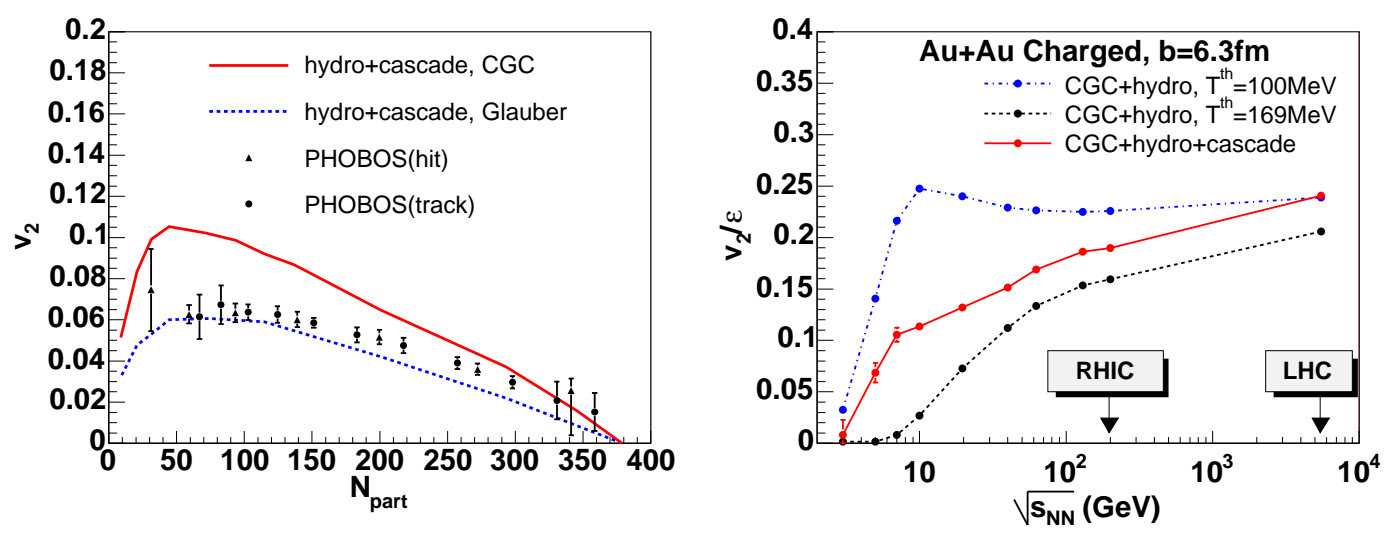

Figure 2. (Left) Centrality dependence of $v_{2}$. The solid (dashed) line results from CGC (Glauber model) initial conditions followed by ideal fluid QGP dynamics and a dissipative hadronic cascade. The data are from the PHOBOS Collaboration [12. (Right) Excitation function of $v_{2} / \varepsilon$ in $b=6.3 \mathrm{fm} \mathrm{Au+Au}$ collisions. The solid line results from CGC initial conditions followed an ideal QGP fluid and a dissipative hadronic cascade. The dashed (dash-dotted) line results from purely ideal fluid dynamics with thermal freezeout at $T^{\text {th }}=169 \mathrm{MeV}(100 \mathrm{MeV})$.

CGC model, however, lead to larger elliptic flows which overshoot the data even after hadronic dissipation is accounted for [3], unless one additionally assumes significant shear viscosity also during the early QGP stage.

Fig. 2 (right) shows the excitation function of the charged particle elliptic flow $v_{2}$, scaled by the initial eccentricity $\varepsilon$, for $\mathrm{Au}+\mathrm{Au}$ collisions at $b=6.3 \mathrm{fm}$ impact parameter, using three different models: (i) a pure 3D ideal fluid approach with a typical kinetic freezeout temperature $T^{\text {th }}=100 \mathrm{MeV}$ where both QGP and hadron gas are treated as ideal fluids (dash-dotted line); (ii) 3D ideal fluid evolution for the QGP, with kinetic freezeout at $T^{\text {th }}=169 \mathrm{MeV}$ and no hadronic rescattering (dashed line); and (iii) 3D ideal fluid QGP evolution followed by hadronic rescattering below $T^{\mathrm{sw}}=169 \mathrm{MeV}$ (solid line). Although applicability of the CGC model for SPS energies might be questioned, we use it here as a systematic tool for obtaining the energy dependence of the hydrodynamic initial conditions. By dividing out the initial eccentricity $\varepsilon$, we obtain an excitation function for the scaled elliptic flow $v_{2} / \varepsilon$ whose shape should be insensitive to the fact that CGC initial conditions produce larger eccentricities and the resulting integrated $v_{2}$ overshoots the data at RHIC. Fig. 22 shows the well-known bump in $v_{2} / \varepsilon$ at SPS energies $\left(\sqrt{s_{N N}} \sim 10 \mathrm{GeV}\right)$ predicted by the purely hydrodynamic approach, as a consequence of the softening of the equation of state (EOS) near the quark-hadron phase transition region [13], and that this structure is completely washed out by hadronic dissipation [14], consistent with the experimental data [15, 16]. Even at RHIC energy, hadronic dissipation still reduces $v_{2}$ by $\sim 20 \%$. The hybrid model predicts a monotonically increasing excitation function for $v_{2} / \varepsilon$ which keeps growing from RHIC to LHC energies [14, contrary to the ideal fluid approach whose excitation function almost saturates above RHIC energies. 
In summary, we have developed a unified dynamical model, based on fully 3D ideal QGP fluid dynamics followed by a realistic hadron cascade, to describe the spacetime evolution of bulk matter in relativistic heavy ion collisions. With Glauber initial conditions, hadronic dissipation seems to be able to reduce the elliptic flow enough to bring theoretical predictions in line with the data at all collision centralities and rapidities, leaving little room for additional dissipative effects in the QGP. CGC initial conditions, however, yield significantly more eccentric sources and produce larger elliptic flow than observed, even if late stage hadronic dissipation is accounted for. To answer the question whether the QGP is a perfect fluid, further systematic studies of the hadron distributions for a variety of species, collision centralities, center of mass energies and system sizes are needed. Our analysis also points to a need for a better understanding of the initial conditions in heavy ion collisions if one hopes to use experimental data to constrain the QGP viscosity (see recent studies of the source eccentricity from classical Yang Mills simulations with a "universal" saturation scale [17] and of effects of eccentricity fluctuation [9]). Our approach predicts that the response of the system to the initial spatial anisotropy of the produced matter, $v_{2} / \varepsilon$, continues to increase towards LHC energies, and that hadronic dissipation washes out the phase-transition bump predicted in [13] and leads to a monotonically excitation function for $v_{2} / \varepsilon$.

This work was supported by the U.S. DOE under contracts DE-FG02-01ER41190 (U.H.), DE-AC02-98CH10886 (D.K.) and DE-FG02-87ER40331.A008 (R.L.).

\section{References}

[1] Back B B et al (PHOBOS Collaboration) 2005 Nucl. Phys. A 75728

Adams J et al (STAR Collaboration) 2005 Nucl. Phys. A 757102

Adcox K et al (PHENIX Collaboration) 2005 Nucl. Phys. A 757184

[2] Kolb P F and Heinz U 2004 in Quark-Gluon Plasma 3 p 634 (edited by Hwa R C and Wang X N, World Scientific, Singapore) (Preprint nucl-th/0305084)

[3] Hirano T, Heinz U, Kharzeev D, Lacey R and Nara Y 2006 Phys. Lett. B 636299

[4] Hirano T 2001 Phys. Rev. C 65011901

[5] Hirano T and Tsuda K 2002 Phys. Rev. C 66054905

[6] Hirano T and Nara Y 2004 Nucl. Phys. A $\mathbf{7 4 3} 305$

[7] Nara Y, Otuka N, Ohnishi A, Niita K and Chiba S 2000 Phys. Rev. C 61024901

[8] Back B B et al (PHOBOS Collaboration) 2002 Phys. Rev. C 65061901

[9] Miller M and Snellings R 2003 Preprint nucl-ex/0312008

Zhu X I, Bleicher M and Stoecker B 2005 Phys. Rev. C 72064911

Andrade R, Grassi F, Hama F, Kodama T and Socolowski Jr O 2006 Phys. Rev. Lett. 97202302

Drescher H J and Nara Y 2006 Preprint nucl-th/0611017

[10] Adil A, Drescher H J, Dumitru A, Hayashigaki A and Nara Y 2006 Phys. Rev. C 74044905

[11] Kolb P F, Huovinen P, Heinz U and Heiselberg H 2001 Phys. Lett. B 500232

[12] Back B B et al (PHOBOS Collaboration) 2005 Phys. Rev. C 72051901

[13] Kolb P F, Sollfrank J and Heinz U 2000 Phys. Rev. C 62054909

[14] Teaney D, Lauret J and Shuryak E V 2001 nucl-th/0110037

[15] Alt C et al (NA49 Collaboration) 2003 Phys. Rev. C 68034903

[16] Adler C et al (STAR Collaboration) 2002 Phys. Rev. C 66034904

[17] Lappi T and Venugopalan R 2006 Phys. Rev. C74 054905 